\title{
Theoretical Inversion of Amino Acids (Alanine and Aspartic Acid) by Semi-empirical Methods
}

\author{
Musa E. Mohamed \\ Department of Chemistry, College of Applied and Industrial Sciences, University of Bahri, \\ Khartoum State, Khartoum, Sudan \\ E-mail address: musa.elballa@gmail.com
}

\begin{abstract}
The inversion reaction coordinate of free amino acids (alanine, aspartic acid) have been computationally calculated by semi-empirical methods AM1. A transition state for free alanine and aspartic acid were obtained as a three membered ring in which the $\alpha-\mathrm{C}-\mathrm{H}$ and $\alpha-\mathrm{C}_{-} \mathrm{CH}_{3}$ are slightly elongated, 1.2 and $2.17 \AA$ respectively in the alanine transition state. The activation energy of alanine is $77.52 \mathrm{kcal} / \mathrm{mol}$ in the gas phase and $76.66 \mathrm{kcal} / \mathrm{mol}$ in aqueous phase, and for aspartic acid is 54.87 $\mathrm{kcal} / \mathrm{mol}$ in the gas phase and $50.86 \mathrm{kcal} / \mathrm{mol}$ in aqueous phase.
\end{abstract}

Keywords: Inversion; alanine; aspartic; semi-empirical methods; AM1

\section{INTRODUCTION}

All living organisms are composed predominantly of $l$-amino acids; however, the origin and the development of $l$-amino acids have not been clarified. Once $l$-amino acids were established, $d$-amino acid was excluded from living systems. $d$-aspartic acid ( $d$-Asp), was detected in various human tissue samples, such as $\operatorname{tooth}^{(1)}$, eye lenses ${ }^{(2)}$ and aorta $^{(3)}$ from elderly individuals.

The presence of $d$-Asp in aged human tissues has been explained as the result of racemization of aspartyl residues in the protein over time in as much as the proteins in such tissues are metabolically inert. Previous studies showed the presence of $d$-Asp in whole tissues. The specific sites of $d$-Asp residues in the lens protein have been found and the mechanism of formation of $d$-Asp in a specific lens protein was proposed ${ }^{(3)}$.

Amino acid racemization (AAR) is used to determine relative dates of biological materials such as bone, shell and teeth and has been used in an archaeological context for over 30 years. During this time a number of significant results have been generated but many have been questioned and the technique remains controversial ${ }^{(4)}$.

Amino acids are the building blocks of protein and most can exist in two different forms levorotatory $(l)$ and dextrorotatory $(d)$, these forms are called enantiomers. In living organisms the amino acids in protein are almost exclusively $l$ and the $d / l$ ratio approaches zero. On aging and after death proteins break down and the $d$ and $l$ forms start to inter convert. This process is called racemization. In time the $d / l$ ratio approaches one. If the $d / l$ ratio is less than one it may be possible to use it to estimate age ${ }^{(4)}$. 
Racemization is the interconversion of the $l$ and $d$ enantiomers of a compound with one chiral centre. The racemization of aspartic acid (Figure 1) is an example of this. In the case of interconversion at the $\alpha$-carbon of an amino acid with two chiral centres such as isoleucine the term epimerization is used. The terms racemization and epimerization are sometimes used interchangeably in the AAR literature with the racemization of aspartic acid and the epimerization of isoleucine being the two reactions most widely used in AAR dating studies $^{(4)}$.<smiles>[NH3+][C@@H](C(=O)[O-])C(=O)O</smiles>

$l$ - aspartic acid

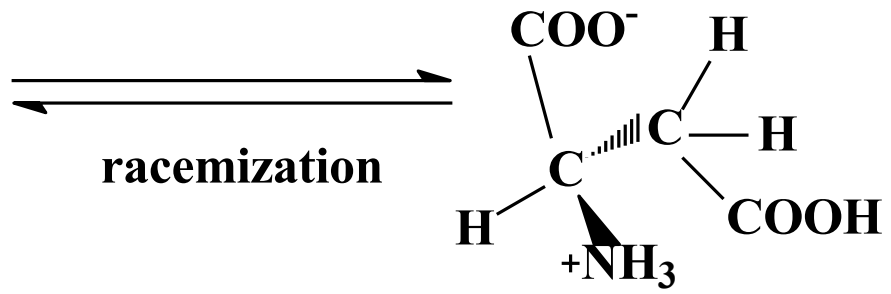

$d$ - aspartic acid

Figure 1. Racemization of Aspartic Acid.

Racemization is a chemical reaction and a number of factors influence its rate ${ }^{(5)}$. These include; amino acid structure, the sequence of amino acids in peptides, $\mathrm{pH}$, buffering effects, metallic cations, the presence of water and temperature. To establish a dating method the kinetics and mechanisms of the racemization (and epimerization) reaction of free and peptide bound amino acids need to be established. In aqueous solutions, racemization rates of amino acids are influenced by; molecular structure, $\mathrm{pH}$, ionic strength, buffer concentration and temperature ${ }^{(5,6,7)}$. The generally accepted mechanism for racemization of amino acids in aqueous solutions invokes a carbanion intermediate (Ebbers et al. ${ }^{(8)}$ ).

This was first postulated by Neuberger ${ }^{(9)}$ and the reaction mechanism involves the removal of the hydrogen bonded to the $\alpha$-carbon by a base such as an hydroxide ion or a water molecule. Subsequently a negatively charged planar carbanion intermediate is formed. The last step is the readdition of a hydrogen ion to the carbanion and there is an equal probability of either the $d$ or the $l$ enantiomer being formed.

Figure 2 illustrates this mechanism. Kinetic studies carried out on $l$-isoleucine in deuterated water (Bada and Schroeder ${ }^{(6)}$ ) is one of many studies that supports the carbanion mechanism. The groups bonded to the $\alpha$-carbon of a free amino acid influence the rate at which racemization occurs. Groups that stabilise the carbanion charge accelerate racemization and groups that destabilise this charge retard racemization (Smith and Evans ${ }^{(7)}$ ). In the zwitterionic form the $-\mathrm{COO}^{-}$group of an amino acid will destabilise the carbanion because of the proximity of two negative charges. 


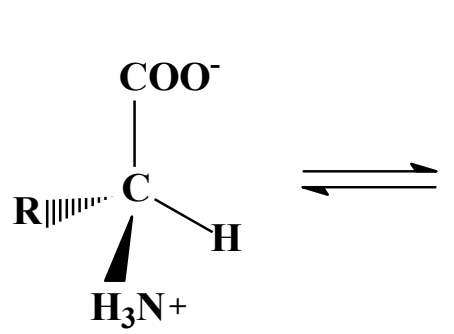

$l$-isomer

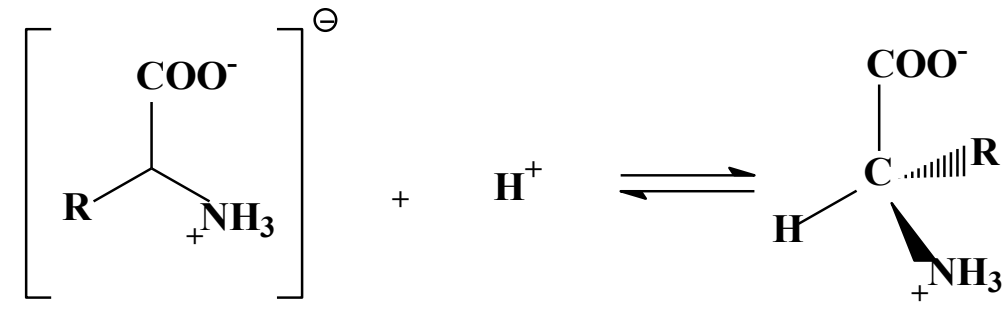

$d$-isomer

Figure 2. The Mechanism for the Racemization of Generalized Amino Acids.

The $-\mathrm{NH}_{3}{ }^{+}$group on the other hand will stabilise the carbanion because of the proximity of opposite charges. Since all free amino acids have a $-\mathrm{COO}^{-}$group and an $-\mathrm{NH}_{3}{ }^{+}$group the characteristics of the different $\mathrm{R}$ groups are the most important in determining the rate of racemization.

The greater the electron withdrawing capacity of the R group of an amino acid the more stable the carbanion will be and the faster the racemization rate will be (Bada and Shou ${ }^{(10)}$; Bada $^{(11)}$; Liardon and Lederman ${ }^{(12)}$ ). Smith and Evans ${ }^{(7)}$ added that the overall effect of an R group depends on the combination of its electron withdrawing or donating characteristics (electronic factors) and its bulk and shape (steric factors). Bulk can hinder proton removal and thus slow the rate.

Based on earlier studies Smith et al. ${ }^{(13)}$ and (Wonnacott ${ }^{(14)}$ ) as cited by (Smith and Evans $\left.{ }^{(7)}\right)$, reported the following order for the rate of racemization of free amino acids in aqueous solution $\mathrm{Ser}>\mathrm{Thr}>\mathrm{Asp}>\mathrm{Phe}>\mathrm{Ala}>\mathrm{Glu}>\mathrm{Leu}>\mathrm{Ile}>$ Val.

\section{METHODS OF CALCULATION}

The initial input was drawn by chemsketch for all amino acids and primary optimized by molecular mechanics using ACDLABS 10 program. Full geometry optimization calculation was carried out by MOPC2007 program, the (Am1, Geo-OK, Precise, Pulay, Gnorm $=0.01)$ keywords request the geometry optimization be performed.

The optimized structures of $l$ and $d$-amino acids were used as inputs (reactants and products) for the calculation of saddle points and transition state, using (saddle, ts) keywords, the transition state was confirmed by only one negative frequency, each transition state was verified to connect to reactants and products by performing an intrinsic reaction coordinate (IRC) analysis.

\section{RESULTS AND DISCUSSION}

The transition state of alanine and aspartic acid was found directly by inverting $l$-amino acid to $d$-amino acid. According to the results of saddle point and transition state, the mechanism of inversion from $l$-to $d$-form in the case of alanine can be postulated as follows: 
in the saddle point structure (Figure 3, Scheme 1) the hydrogen (H7) is inverted in the plane of the chiral carbon (C3) and methyl carbon (C5) to form three-membered ring with them.

Partial double bonds form between $\mathrm{C} 3$ and $\mathrm{C} 5, \mathrm{C} 3$ and N4, C1 and $\mathrm{O} 2$, the length of these bonds is $1.26,1.28$ and $0.87 \AA$ respectively (Scheme 1, Table 1).

In the transition state structure (Figure 3 , Scheme 1), the three membered ring is distorted and the bonds between $\mathrm{C} 3, \mathrm{C} 5$ and $\mathrm{H} 7$ slightly elongated, C3-C5 equal $2.17 \AA$, C5$\mathrm{H} 7=1.47 \AA$ and $\mathrm{C} 3-\mathrm{H} 7=1.2 \AA$, the hydrogen atom $(\mathrm{H} 7)$ moved into the other side of the molecule, the final structure ( $d$-form), differ from the first structure in the position of hydrogen.

The calculated free energy of activation of transition state $\Delta \mathrm{G}^{\ddagger}$ (the energy required for $l$-alanine to achieve the transition state) was found to be $77.519 \mathrm{kcal} / \mathrm{mol}$, and for aspartic acid is $54.87 \mathrm{kcal} / \mathrm{mol}$ in the gas phase (Table 2), and in aqueous phase was found to be 76.66 and $50.86 \mathrm{kcal} / \mathrm{mol}$ for alanine and aspartic respectively. The structures of $l$-aspartic acid, saddle point, transition state and $d$-asparic acid are shown in Figure (4).

Table 1. Bond length for the structures of the reaction path from reactants to products in Angstrom.

\begin{tabular}{lllll}
\hline Bond length in $\AA$ & L-alanine & $\begin{array}{l}\text { Saddle } \\
\text { point }\end{array}$ & $\begin{array}{l}\text { Transition } \\
\text { state }\end{array}$ & D-alnine \\
\hline $\mathrm{H} 7-\mathrm{C} 3$ & 1.13 & 1.01 & 1.20 & 1.13 \\
$\mathrm{C} 5-\mathrm{C} 3$ & 1.52 & 1.26 & 2.17 & 1.52 \\
$\mathrm{~N} 4-\mathrm{C} 3$ & 1.44 & 1.28 & 1.33 & 1.44 \\
$\mathrm{C} 1-\mathrm{C} 3$ & 1.52 & 1.44 & 1.50 & 1.52 \\
$\mathrm{C} 1-\mathrm{O} 2$ & 1.23 & 0.87 & 1.22 & 1.23 \\
$\mathrm{C} 5-\mathrm{H} 7$ & ------ & 1.00 & 1.47 & ----- \\
& & & & \\
\hline
\end{tabular}

According to these results the inversion of amino acids is more favorable in aqueous phase than in the gas phase. The inversion of aspartic acid is more faster than alanine according to the presence of the group with greater electron withdrawing capacity in aspartic acid $\left(-\mathrm{CH}_{2} \mathrm{COOH}\right)^{(10,11 \& 12)}$, which facilitate the stability of the carbanion and will be the faster the racemization rate.

Table 2. Calculated Energies of activation of transition states of alanine and aspartic acid in gas phase and in aqueous phase using Semi-empirical Method AM1 in Kcal/mol.

\begin{tabular}{cll}
\hline Compound & $\begin{array}{c}\Delta \mathrm{G}^{\dagger} \text { in gas phase } \\
\mathrm{kcal} / \mathrm{mol}\end{array}$ & $\begin{array}{l}\Delta \mathrm{G}^{\ddagger} \text { in aqueous phase } \\
\mathrm{kcal} / \mathrm{mol}\end{array}$ \\
\hline alanine transition state & 77.52 & 76.66 \\
aspartic transition state & 54.87 & 50.86 \\
& & \\
\hline
\end{tabular}



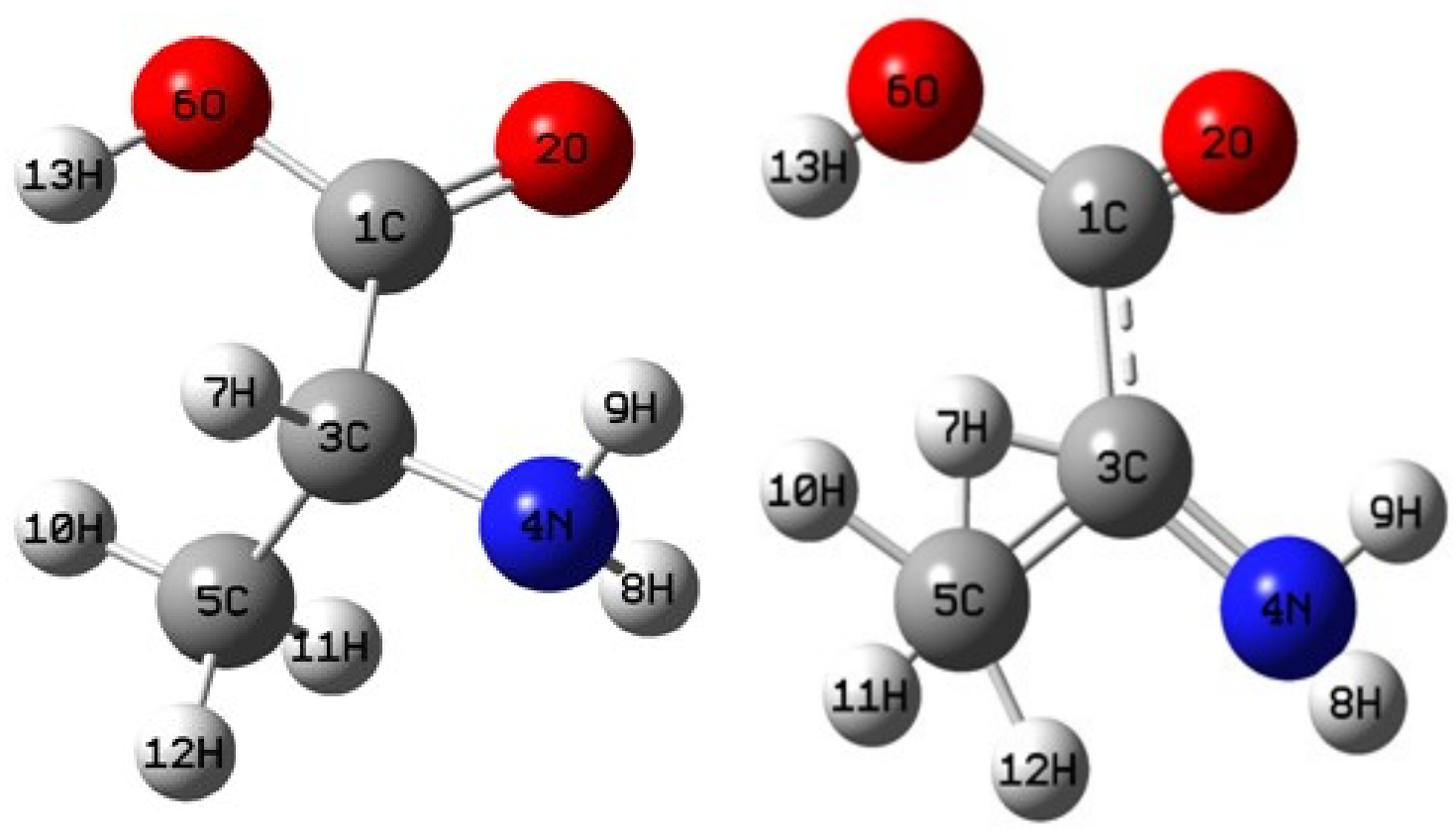

l-alanine

alanine-saddle point
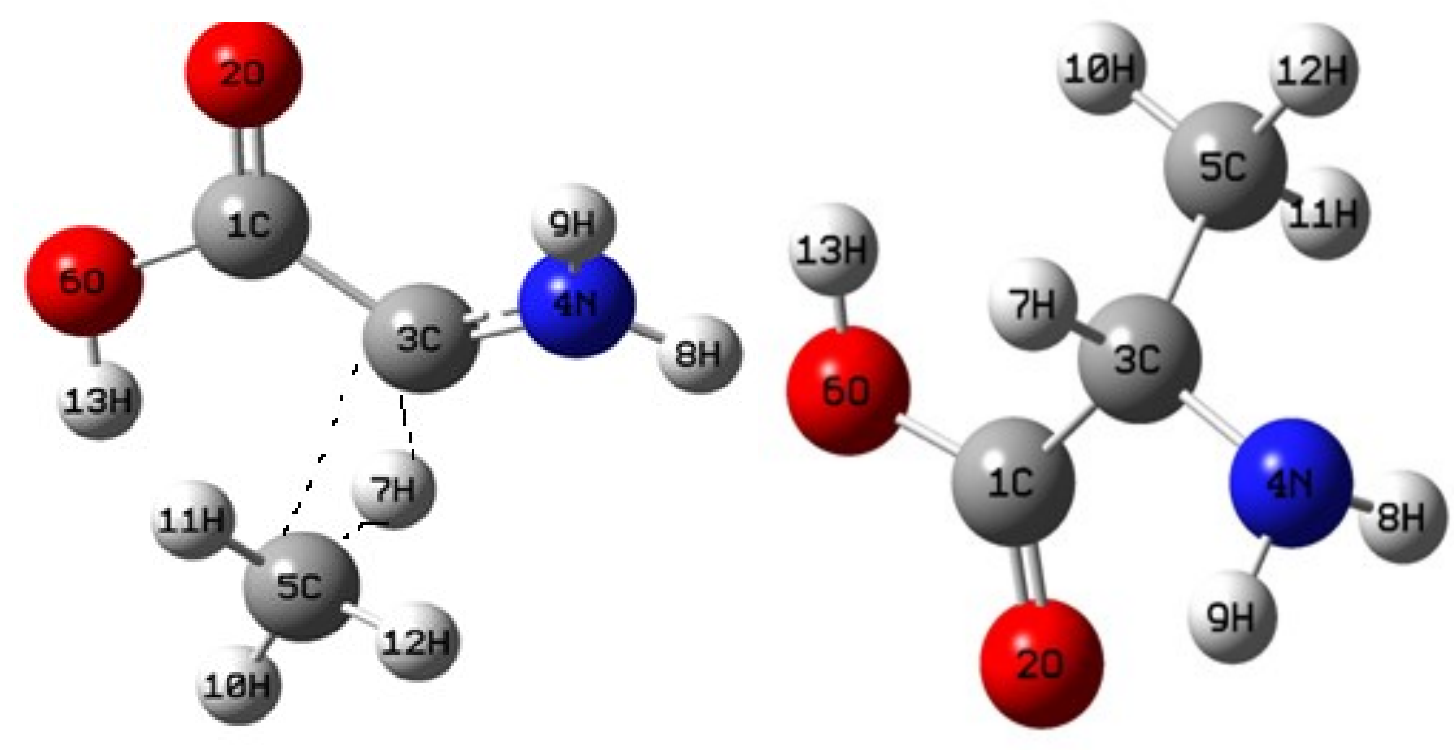

alanine -transition state

$d$-alanine

Figure 3. Structures of $l$-Alanine, Saddle Point, Transition State and $d$-Alanine by Semiemprical Methods. 
<smiles>C=C(O)C(C)N</smiles>

$l$-alanine

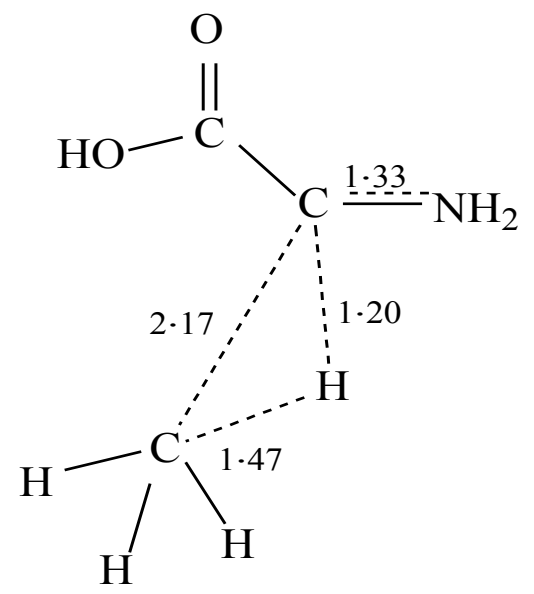

alanine - transition state<smiles>[4H][14CH2][14CH]([14CH2]N)C(=O)O</smiles>

alanine - saddle point<smiles>CC(N)C(=O)O</smiles>

d-alanine

Scheme 1. Proposed Pathway Mechanism of Direct Inversion of $l$-Alanine to $d$-Alanine by Semiempirical Method AM1.

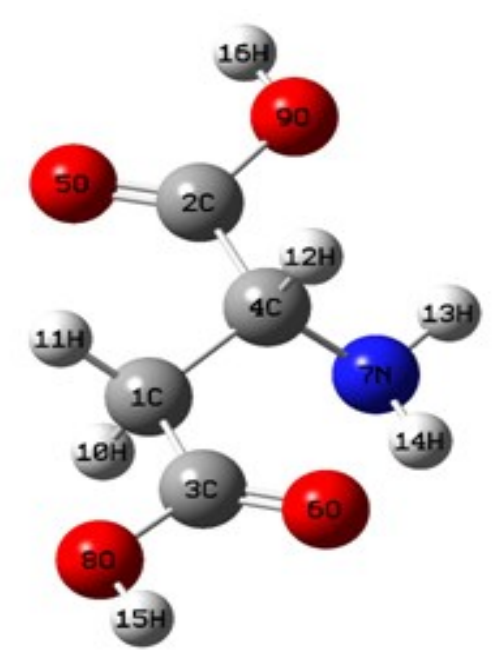

$l$-aspartic acid

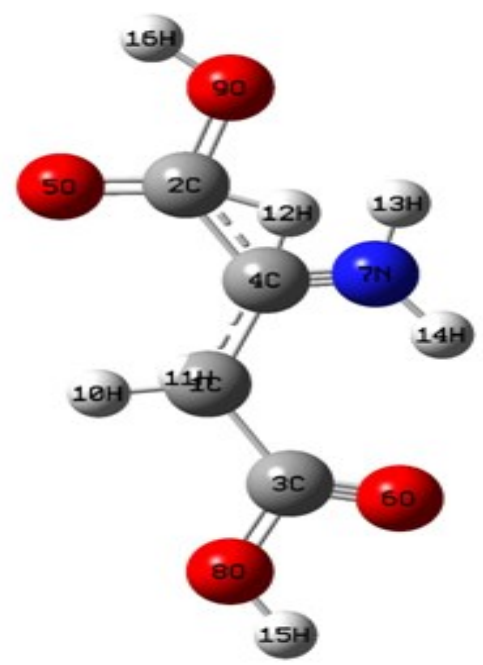

aspartic acid-saddle point 


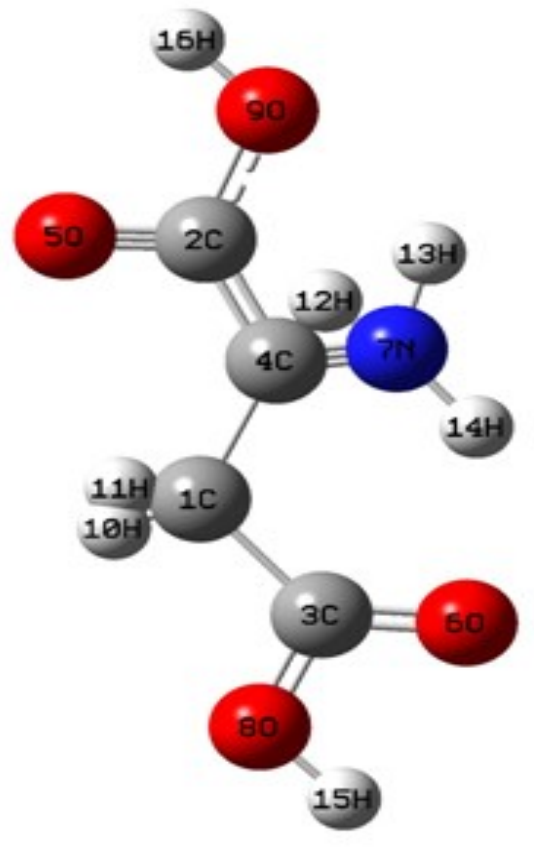

Aspartic acid-transition state

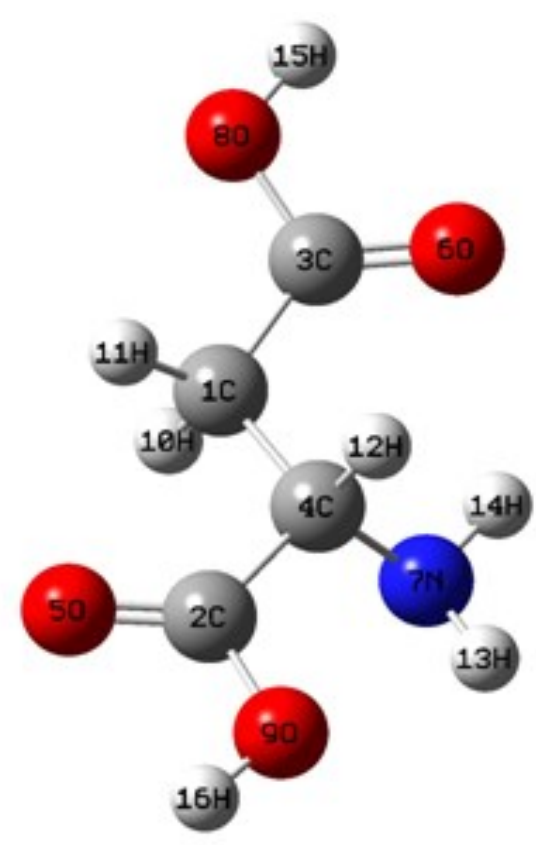

$d$--aspartic acid

Figure 4. Structures of $l$-Aspartic Acid, Saddle Point, Transition State and $d$-Aspartic Acid by Semiempirical Methods AM1.

\section{CONCLUSION}

The direct inversion of free amino acids (alanine, aspartic acid) have been computationally calculated by semi-empirical methods AM1 using MOPAC2007, and a three membered ring transition state was obtained.

The inversion of amino acids is more favorable in aqueous phase than in the gas phase. The electron withdrawing substituent groups increase the rate of inversion.

\section{SUPPLEMENTARY INFORMATIONS}

The supplementary informations (output results of semi-empirical calculations) are available as free of charge on request.

\section{References}

[1] Helfman P. M., Bada, J. L., Nature 262 (1976) 279-28.

[2] Masters P. M., Bada J. L., Zigler J. S. Jr., Nature 268 (1977) 71-73.

[3] Powell J. T., Vine N., Crossman M., Atherosclerosis 97 (1992) 201-208.

[4] Johnson B. J., Miller G. H., Archaeometry 39(2) (1997) 265-287. 
[5] Rutter N. W., Blackwell B., Geological Association of Canada (1995) 125-164.

[8] Bada J. L., Schroeder R. A., Die Naturwissenschaften 62 (1975) 71-79.

[7] Smith G. G., Evans R. C., Biogeochemistry of Amino Acids, New York: John Wiley and Sons. (1980) 257-282.

[8] Ebbers E. J., Ariaans G. J. A., Houbiers J. P. M., Bruggink A., Zwanenburg B, Tetrahedron 53 (1997) 9417-9476.

[9] Neuberger A., Advances in Protein Chemistry, New York: Academic Press, 4 (1948) 297-383

[10] Bada J. L., Shou M. Y., Biogeochemistry of Amino Acids, New York, John Wiley and Sons, (1980) 235-255.

[11] Bada J. L., Interdisciplinary, Science Reviews 7(1) (1982) 30-46.

[12] Liardon R., Ledermann S., Journal of Agricultural and Food Chemistry 34 (1986) 557-565.

[13] Smith G. G., Williams K. M., Wonnacott D. M., The Journal of Organic Chemistry, 43(1) (1978) 1-5.

[14] Wonnacott D. M., PhD thesis, Utah State University, 1979. 